\title{
On the supersymmetry of the Dirac-Kepler problem plus a Coulomb-type scalar potential in $D+1$ dimensions and the generalized Lippmann-Johnson operator
}

\author{
D. Martínez ${ }^{a *}$ M. Salazar-Ramírez ${ }^{b}$ \\ R. D. Mota $^{c}$ and V. D. Granados ${ }^{b}$
}

${ }^{a}$ Universidad Autónoma de la Ciudad de México, Plantel Cuautepec, Av. La Corona 320, Col. Loma la Palma, Delegación Gustavo A. Madero, 07160, México D. F., México.

${ }^{b}$ Escuela Superior de Física y Matemáticas, Instituto Politécnico Nacional, Ed. 9, Unidad Profesional Adolfo López Mateos, 07738 México D F, México.

${ }^{c}$ Escuela Superior de Ingeniería Mecánica y Eléctrica, Unidad Culhuacán, Instituto Politécnico Nacional, Av. Santa Ana No. 1000, Colonia San Francisco Culhuacán, Delegación Coyoacán, C. P. 04430, México D. F., Mexico.

\begin{abstract}
We study the Dirac-Kepler problem plus a Coulomb-type scalar potential by generalizing the Lippmann-Johnson operator to $D$ spatial dimensions. From this operator, we construct the supersymmetric generators to obtain the energy spectrum for discrete excited eigenstates and the radial spinor for the SUSY ground state.
\end{abstract}

PACS: 03.65.Ge; 03.65.Pm; 11.30.Pb

${ }^{*}$ E-mail address: dmartinezs77@yahoo.com.mx 


\section{Introduction}

The vector potential is introduced into the Dirac equation by minimal coupling, whereas the scalar potential is added to the mass term. Thus, it can be interpreted as a position-dependent mass term. The vector Coulomb potential can be derived from the exchange of massless photons between the nucleus and the leptons orbiting around it, the Coulomb-type potential can be created by exchange of massless scalar mesons [1]. The scalar potential has been of great importance in the relativistic quark model, it was employed for describing magnetic moments and to avoid the Klein paradox risen in the quarkonium confining potentials [2]. Also, a relativistic scalar potential has been used as a model for the spatially dependent valence and conduction band edges of semiconductors near the $\Gamma$ and $L$ points in the Brillouin zone [3, 4].

The Dirac-Kepler problem in $D+1$ dimensions has been treated in several ways: by power series [5], radial SUSY QM [6], supersymmetry generated by the Lippmann-Johnson operator [7] and an $s u(1,1)$ approach [8]. On the other hand, the Dirac radial equations with vector and scalar Coulomb-type potentials were studied by series power [9], SUSY QM [10] and intertwining operators [11].

Joseph was the first who studied the Dirac equation with vector potential in $D$ spatial dimensions [12]. Also, the Lippmann-Johnson operator was introduced to generate the supersymmetric charges [13]. With Coulombtype scalar and vector potentials, the problem was solved in analytical way by reducing the radial Dirac equations to the differential equations satisfied by confluent hypergeometric functions [2, 14. The energy spectrum and the SUSY ground state of this problem were found by an $s u(1,1)$ algebraic approach [15]. Notice that in order to the Coulomb potential obeys the Gauss' law, it must be of the form $-\alpha / r^{D-2}$. However, it has been shown that this potential leads to unstable orbits at the classical regime [16, 17] whereas for the non-relativistic quantum treatment for bound states there are not normalizable wave functions [18]. These features have compelled to set the Coulomb potential in higher dimensions as the $-\alpha / r$ potential [2, 13, 14, 15. In the present work we restrict the vector and scalar potentials to have this form. To our knowledge the Lippmann-Johnson operator in general dimensions with both potentials has not been reported and as a consequence, the supersymmetry generated from this constant of motion remains untreated

The purpose of this Letter is to construct the supersymmetry charges for 
the Dirac-Kepler problem plus a Coulomb scalar potential from the generalized Lippmann-Johnson operator in $D$ dimensions. The fact that one of the supercharges annihilates the SUSY ground state leads us to find the energy spectrum for discrete excited states and to obtain the radial differential equations for the SUSY ground state. By performing a similarity transformation to the radial Lippmann-Johnson operator we find the SUSY ground state. Also, we show that the radial part of the Lippmann-Johnson operator is reduced to that reported in [11] for the three-dimensional space and obtained by intertwining considerations.

\section{The relativistic Dirac equation in $D+1$ di- mensions and the generalized Lippmann- Johnson operator}

The Dirac equation in $D+1$ dimensions for a central field can be written with $(\hbar=c=1)$ as 9 ]

$$
H \Psi \equiv\left\{\gamma^{0} \gamma^{j} p^{j}+\gamma^{0}\left(m+V_{s}(r)\right)+V_{v}(r)\right\} \Psi=i \frac{\partial \Psi}{\partial t}, \quad j=1,2, \ldots, D
$$

where summation over repeated index is assumed, $m$ is the mass of the particle, $V_{s}$ and $V_{v}$ are the spherically symmetric scalar and vector potentials, respectively, and the Dirac matrices in $D$ dimensions, $\gamma^{j}$, satisfy $\left\{\gamma^{\mu}, \gamma^{\nu}\right\}=$ $2 \eta^{\mu \nu}$ with

$$
\eta^{\mu \nu}= \begin{cases}\delta^{\mu \nu} & \text { if } \mu=0 \\ -\delta^{\mu \nu} & \text { if } \mu \neq 0\end{cases}
$$

In $D$ spatial dimensions, the orbital angular momentum operator $L_{a b}$ and the total angular momentum $J_{a b}$ are defined as

$$
L_{a b}=i x_{a} \partial_{b}-i x_{b} \partial_{a}
$$

and

$$
J_{a b}=L_{a b}+\frac{i}{2} \gamma^{a} \gamma^{b}
$$

respectively. In the case of spherically symmetric potentials, the total angular momentum operator and the spin-orbit operator

$$
K_{D}=-\gamma^{0}\left\{\frac{i}{2} \sum_{a \neq b} \gamma^{a} \gamma^{b} L_{a b}+\frac{1}{2}(D-1)\right\}
$$


commute with the Dirac Hamiltonian. For a given total angular momentum $j$, the eigenvalues of $K_{D}$ are $\kappa_{D}= \pm(j+1 / 2)$, where the minus sign is for aligned spin $j=\ell+\frac{1}{2}$, and the plus sign is for unaligned spin $\ell-\frac{1}{2}$ [14].

We find that if the scalar and vector potentials are given by $V_{s}=\frac{\alpha_{s}}{r}$ and $V_{v}=\frac{\alpha_{v}}{r}$ then, the matrix hermitian operator

$$
B=-i K_{D} \gamma^{D+1}\left(H-\gamma^{0} m\right)+\gamma^{D+1} \gamma^{0} \gamma^{i} \frac{x^{i}}{r}\left(\alpha_{v} m+\alpha_{s} H\right)
$$

is a constant of motion (see appendix A), where the pseudoscalar $\gamma^{D+1}$ is reduced to the matrix $\gamma^{5}$ in $(3+1)$ dimensions and satisfies $\left(\gamma^{D+1}\right)^{\dagger}=\gamma^{D+1}$, $\left(\gamma^{D+1}\right)^{2}=1$ and $\left\{\gamma^{D+1}, \gamma^{\mu}\right\}=0$. Also, it can be shown that this operator anticommutes with the Dirac operator $K_{D}$. Therefore, the operator $B$ satisfies

$$
B \Psi_{\kappa_{D}}=-b \Psi_{-\kappa_{D}},
$$

where $b$ is an undetermined constant. In fact, $B$ is the generalization of the Lippmann-Johnson operator [19] and is reduced to that given in [11] for $D=3$, and to that for $D$ dimensions in absence of the scalar potential $V_{s}$ reported in [13].

In this way, for odd or even dimensions, we write the eigenstates of equation (1) as

$$
\Psi_{\kappa_{D}}=r^{-\frac{D-1}{2}}\left(\begin{array}{c}
G_{\kappa_{D}}^{(1)}(r) \chi_{\kappa_{D}}^{\mu}\left(\Omega_{D}\right) \\
i G_{\kappa_{D}}^{(2)}(r) \chi_{-\kappa_{D}}^{\mu}\left(\Omega_{D}\right)
\end{array}\right) e^{-i E t}
$$

being $G_{\kappa_{D}}^{(1)}$ and $G_{\kappa_{D}}^{(2)}(r)$ the radial functions, and $\chi_{\kappa_{D}}^{\mu}\left(\Omega_{D}\right)$ the hyperspherical harmonic functions coupled with the angular momentum $j$ [14]. We consider that

$$
\gamma^{D+1} \gamma^{0} \gamma^{i} \frac{x^{i}}{r} \Psi_{\kappa_{D}}=-\Psi_{-\kappa_{D}}
$$

which is the generalization of the three-dimensional equation $(\vec{\sigma} \cdot \hat{r}) \Psi_{\kappa}=$ $-\Psi_{-\kappa}[7,21]$. By defining the spinors

$$
\Theta_{\kappa_{D}}=\left(\begin{array}{c}
-G_{\kappa_{D}}^{(2)} \chi_{\kappa_{D}} \\
i G_{\kappa_{D}}^{(1)} \chi_{-\kappa_{D}}
\end{array}\right) e^{-i E t}, \Phi_{\kappa_{D}}=\left(\begin{array}{c}
G_{\kappa_{D}}^{(1)} \chi_{\kappa_{D}} \\
i G_{\kappa_{D}}^{(2)} \chi_{-\kappa_{D}}
\end{array}\right) e^{-i E t}
$$

and from the results of Appendix $\mathrm{B}$, the explicit form of the operator $B$ 
acting on a general eigenstate of the Dirac Hamiltonian is

$$
\begin{aligned}
& B \Psi_{\kappa_{D}}=-r^{-\frac{D-1}{2}}\left\{\left(\alpha_{s} \partial_{r}-\kappa_{D} V_{v}\right) \Theta_{\kappa_{D}}+\left(\kappa_{D}\left(\partial_{r}+\frac{\kappa_{D}}{r} \gamma^{0}\right)+m \alpha_{v}\right) \Phi_{\kappa_{D}}\right. \\
& \left.+\alpha_{s} \gamma^{0}\left(m+V_{s}+\gamma^{0} V_{v}\right) \Phi_{\kappa_{D}}\right\} \text {. }
\end{aligned}
$$

\section{SUSY QM and the energy spectrum}

Based on references [7, 13, 20], we define the supersymmetric generator

$$
Q=\left(\begin{array}{cc}
0 & 0 \\
B & 0
\end{array}\right)
$$

which satisfies $\{Q, Q\}=0$ and

$$
\mathcal{H} \equiv\left\{Q, Q^{\dagger}\right\}=\left(\begin{array}{cc}
B^{2} & 0 \\
0 & B^{2}
\end{array}\right)
$$

with $\mathcal{H}$ the supersymmetric Hamiltonian.

In order to obtain the energy spectrum for the Dirac Hamiltonian $H$, we consider the results given in Appendix C, from which

$$
B^{2}=\left(\alpha_{v} m+\alpha_{s} H\right)^{2}+K_{D}^{2}\left(H^{2}-m^{2}\right) .
$$

By considering equation (7), we have the eigenvalue equation $B^{2} \Psi_{\kappa_{D}}=$ $b^{2} \Psi_{\kappa_{D}}$. Thus

$$
b^{2}=\left(\alpha_{v} m+\alpha_{s} E\right)^{2}+\kappa_{D}^{2}\left(E^{2}-m^{2}\right) .
$$

Since the supersymmetric ground state, $\Psi_{S U S Y}^{0}$ must satisfy the condition

$$
\mathcal{H} \Psi_{S U S Y}^{0}=0
$$

it follows that the ground state energy eigenvalue, $E_{0}$, is obtained from equation (15) by setting $b=0$. In this way

$$
E_{0}=m\left\{-\frac{\alpha_{s} \alpha_{v}}{\alpha_{v}^{2}+\gamma^{2}} \pm \sqrt{\left(\frac{\alpha_{s} \alpha_{v}}{\alpha_{v}^{2}+\gamma^{2}}\right)^{2}-\left(\frac{\alpha_{s}^{2}-\gamma^{2}}{\alpha_{v}^{2}+\gamma^{2}}\right)}\right\}
$$


where $\gamma^{2}=\kappa_{D}^{2}+\alpha_{s}^{2}-\alpha_{v}^{2}$. For the excited states of the Hamiltonian $H$, we perform the change $\gamma \rightarrow \gamma+n$, where $n=0,1,2,3 \ldots$ is the radial quantum number. Then

$$
\frac{E_{n}}{m}=-\frac{\alpha_{s} \alpha_{v}}{\alpha_{v}^{2}+(\gamma+n)^{2}} \pm \sqrt{\left(\frac{\alpha_{s} \alpha_{v}}{\alpha_{v}^{2}+(\gamma+n)^{2}}\right)^{2}-\left(\frac{\alpha_{s}^{2}-(\gamma+n)^{2}}{\alpha_{v}^{2}+(\gamma+n)^{2}}\right)}
$$

which is in accordance to that obtained from an analytical [9, 14, or $s u(1,1)$ algebraic approach [15].

The eigenstates $\Psi_{\kappa_{D}}$ and $\Psi_{-\kappa_{D}}$ are transformed into each other by the operator $B$ (equation (7D) ) and both are eigenfuntions of the operator $B^{2}$ with the same eigenvalue. Therefore, the supersymmetric eigenstates can be written as

$$
\Psi_{S U S Y}=\left(\begin{array}{c}
\Psi_{\kappa_{D}} \\
\Psi_{-\kappa_{D}}
\end{array}\right)
$$

Considering equations (13) and (16), the components of the sypersymmetric ground state must satisfy

$$
B \Psi_{0 \pm \kappa_{D}}=0 .
$$

In order to solve this equation for $+\kappa_{D}$ (the solution for the other sign can be obtained by equation (7)) we consider the expression (B.3), from which

$$
\begin{aligned}
B \Psi_{\kappa_{D}} & =-r^{-\frac{D-1}{2}} e^{-i E t}\left\{\left(\alpha_{s} \partial_{r}-\kappa_{D} V_{v}\right)\left(\begin{array}{c}
-G_{\kappa_{D}}^{(2)} \chi_{\kappa_{D}} \\
i G_{\kappa_{D}}^{(1)} \chi_{-\kappa_{D}}
\end{array}\right)\right. \\
& +\left(\kappa_{D} \partial_{r}+m \alpha_{v}+\alpha_{s} V_{v}\right)\left(\begin{array}{c}
G_{\kappa_{D}}^{(1)} \chi_{\kappa_{D}} \\
i G_{\kappa_{D}}^{(2)} \chi_{-\kappa_{D}}
\end{array}\right) \\
& \left.+\left(\frac{\kappa_{D}^{2}}{r}+\alpha_{s}\left(m+V_{s}\right)\right)\left(\begin{array}{c}
G_{\kappa_{D}}^{(1)} \chi_{\kappa_{D}} \\
-i G_{\kappa_{D}}^{(2)} \chi_{-\kappa_{D}}
\end{array}\right)\right\} .
\end{aligned}
$$

Thus, the radial components of the SUSY ground state $\left(G_{0 \kappa_{D}}^{(1)}\right.$ and $\left.G_{0 \kappa_{D}}^{(2)}\right)$ satisfy

$$
L_{D}\left(\begin{array}{l}
G_{0 \kappa_{D}}^{(1)} \\
G_{0 \kappa_{D}}^{(2)}
\end{array}\right)=\left(\begin{array}{l}
0 \\
0
\end{array}\right)
$$

where

$$
L_{D} \equiv\left(\begin{array}{cc}
\frac{d}{d r}+\frac{\epsilon_{+}}{r}+\frac{m \alpha_{+}}{\kappa_{D}} & -\frac{\alpha_{s}}{\kappa_{D}} \frac{d}{d r}+\frac{\alpha_{v}}{r} \\
\frac{\alpha_{s}}{\kappa_{D}} \frac{d}{d r}-\frac{\alpha_{v}}{r} & \frac{d}{d r}-\frac{\epsilon_{-}}{r}-\frac{m \alpha_{-}}{\kappa_{D}}
\end{array}\right)
$$


$\epsilon_{ \pm}=\kappa_{D}+\alpha_{s} \alpha_{ \pm} / \kappa_{D}$ and $\alpha_{ \pm}=\left(\alpha_{s} \pm \alpha_{v}\right)$. The matrix operator $L_{D}$, obtained by means of SUSY QM, is reduced to the three-dimensional operator $L$ reported in [11], which has been constructed by imposing an intertwining relation between the corresponding radial Dirac Hamiltonian and $L$.

In order to find the expression for the radial components of the SUSY ground state, we define

$$
\left(\begin{array}{l}
\tilde{G}_{0 \kappa_{D}}^{(1)} \\
\tilde{G}_{0 \kappa_{D}}^{(2)}
\end{array}\right)=\left(\begin{array}{cc}
1 & -\frac{\alpha_{s}}{\kappa_{D}} \\
\frac{\alpha_{s}}{\kappa_{D}} & 1
\end{array}\right)\left(\begin{array}{l}
G_{0 \kappa_{D}}^{(1)} \\
G_{0 \kappa_{D}}^{(2)}
\end{array}\right)
$$

Therefore, equation (23) is rewritten as

$$
\left\{\frac{d}{d r}+\frac{1}{r}\left(\begin{array}{cc}
\kappa_{D} & \alpha_{+} \\
\alpha_{-} & -\kappa_{D}
\end{array}\right)\right\}\left(\begin{array}{l}
\tilde{G}_{0 \kappa_{D}}^{(1)} \\
\tilde{G}_{0 \kappa_{D}}^{(2)}
\end{array}\right)=-\frac{m \kappa_{D}}{\kappa_{D}^{2}+\alpha_{s}^{2}}\left(\begin{array}{cc}
\alpha_{+} & \frac{\alpha_{s} \alpha_{+}}{\kappa_{D}} \\
\frac{\alpha_{s} \alpha_{-}}{\kappa_{D}} & -\alpha_{-}
\end{array}\right)\left(\begin{array}{l}
\tilde{G}_{0 \kappa_{D}}^{(1)} \\
\tilde{G}_{0 \kappa_{D}}^{(2)}
\end{array}\right),
$$

which can be easily solved by diagonalizing the matrix of the factor $1 / r$. For this purpose, we perform the transformation

$$
\left(\begin{array}{c}
\tilde{F}_{0 \kappa_{D}}^{(1)} \\
\tilde{F}_{0 \kappa_{D}}^{(2)}
\end{array}\right)=\left(\begin{array}{cc}
\kappa_{D}+\gamma & \alpha_{+} \\
-\alpha_{-} & \kappa_{D}+\gamma
\end{array}\right)\left(\begin{array}{c}
\tilde{G}_{0 \kappa_{D}}^{(1)} \\
\tilde{G}_{0 \kappa_{D}}^{(2)}
\end{array}\right)
$$

Thus, from equation (25), we obtain

$\left\{\frac{d}{d r}+\frac{1}{r}\left(\begin{array}{cc}\gamma & 0 \\ 0 & -\gamma\end{array}\right)\right\}\left(\begin{array}{l}\tilde{F}_{0 \kappa_{D}}^{(1)} \\ \tilde{F}_{0 \kappa_{D}}^{(2)}\end{array}\right)=-\frac{m}{\kappa_{D}^{2}+\alpha_{s}^{2}}\left(\begin{array}{cc}\alpha_{v} \kappa_{D}+\alpha_{s} \gamma & 0 \\ 0 & \alpha_{v} \kappa_{D}-\alpha_{s} \gamma\end{array}\right)\left(\begin{array}{l}\tilde{F}_{0}^{(1)} \\ \tilde{F}_{0 \kappa_{D}}^{(2)}\end{array}\right)$.

The unnormalized solutions for these differential equations are given by

$$
\begin{aligned}
& \tilde{F}_{0 \kappa_{D}}^{(1)}=r^{-\gamma} \exp \left(-\frac{m}{\kappa_{D}^{2}+\alpha_{s}^{2}}\left(\alpha_{v} \kappa_{D}+\alpha_{s} \gamma\right) r\right), \\
& \tilde{F}_{0 \kappa_{D}}^{(2)}=r^{\gamma} \exp \left(-\frac{m}{\kappa_{D}^{2}+\alpha_{s}^{2}}\left(\alpha_{v} \kappa_{D}-\alpha_{s} \gamma\right) r\right) .
\end{aligned}
$$

Since $\tilde{F}_{0 \kappa_{D}}^{(1)}$ diverges at $r=0$, it is not a physically acceptable solution. Hence, the radial spinor for the supersymmetric ground state is

$$
\psi_{S U S Y}^{0} \equiv\left(\begin{array}{c}
0 \\
r^{\gamma} \exp \left(-\frac{m}{\kappa_{D}^{2}+\alpha_{s}^{2}}\left(\alpha_{v} \kappa_{D}-\alpha_{s} \gamma\right) r\right)
\end{array}\right)
$$


For the case $\alpha_{s}=0, \psi_{S U S Y}^{0}$ is a normalizable solution only for $\kappa_{D}<0$ which is in accordance to the results reported in [7] for $3+1$ dimensions.

Having obtained the SUSY ground state, the explicit form of the eigenfunctions corresponding to higher SUSY energy levels should be determined by solving the eigenvalue equation $\mathcal{H} \Psi_{S U S Y}=b^{2} \Psi_{S U S Y}$ which is equivalent to find the solutions of the equation $B^{2} \Psi_{\kappa_{D}}=b^{2} \Psi_{\kappa_{D}}$. Nevertheless, this problem is much more complicated to solve than the original Dirac eigenvalue equation.

It is worth noting that the excited energy levels for the Hamiltonian $H$ could be obtained in a different way by applying the Sukumar [6] or the Thaller [22] approaches for the shape-invariant uncoupled second order Dirac equations. However, in the present Letter we used the generalization of the Lippmann-Johnson operator in $D$ dimensions and SUSY QM to obtain the energy spectrum for this problem.

\section{Concluding remarks}

We treated the Dirac-Kepler problem plus Coulomb-type scalar potential by generalizing the Lippmann-Johnson operator in general dimensions. This operator allowed us to construct the supersymmetric charges from which we found the ground state energy spectrum and the spectrum energy for discrete excited states. The action of the Lippmann-Johnson operator on the ground state of the Dirac equation leads to the radial operator $L_{D}$, equation (23), which generalizes to $D$-dimensions that reported in [11] for the three-dimensional space. The ground state obtained in this work is in full agreement with those reported in [15] and it reduces to that in three dimensions reported in [10]. With the generalized Lippmann-Johnson operator reported in the present Letter, we can construct an $S O(4)$ symmetry treatment, similar to that given for the Kepler-Coulomb problem in three spatial dimensions [23, which is a work in progress. 


\section{A Calculation of $[B, H]=0$}

For an arbitrary radial function $f(r)$, we show that $\left[K_{D}, f(r)\right]=0$. Moreover, we find that

$$
\begin{aligned}
{\left[H, \gamma^{0}\right] } & =-2 \gamma^{i} p^{i}, \\
{\left[H, \gamma^{D+1}\right] } & =-2 \gamma^{D+1} \gamma^{0}\left(m+V_{s}\right), \\
{\left[H, \gamma^{D+1} \gamma^{0} \gamma^{a} \frac{x^{a}}{r}\right] } & =\frac{2 i}{r} \gamma^{D+1} \gamma^{0} K_{D} .
\end{aligned}
$$

Hence,

$$
\begin{aligned}
{[B, H] } & =-i K_{D}\left[H, \gamma^{D+1}\right]\left(H-\gamma^{0} m\right) \\
& +i m K_{D} \gamma^{D+1}\left[H, \gamma^{0}\right] \\
& +\left[H, \gamma^{D+1} \gamma^{0} \gamma^{a} \frac{x^{a}}{r}\right]\left(\alpha_{v} m+\alpha_{s} H\right) \\
& =-2 i K_{D} \gamma^{D+1} \gamma^{0}\left\{\left(m+V_{s}\right)\left(H-\gamma^{0} m\right)\right. \\
& \left.-m \gamma^{0} \gamma^{a} p^{a}-\left(V_{v} m+V_{s} H\right)\right\} \\
& =0 .
\end{aligned}
$$

\section{B Calculation of $B \Psi_{\kappa_{D}}$}

Considering the properties of the operator $K_{D}$, the algebra satisfied by the matrices $\gamma^{i}$ and from equations (9) and (10), we obtain

$$
\begin{aligned}
-i K_{D} \gamma^{D+1}\left(H-\gamma^{0} m\right) \Psi_{\kappa_{D}} & \\
= & i\left\{\gamma^{D+1} \gamma^{0} \gamma^{a} \frac{x^{a}}{r}\left[\frac{x^{b}}{r} p^{b}-\frac{i}{r}\left(\gamma^{0} K_{D}+\frac{D-1}{2}\right)\right]\right. \\
& \left.+\left(V_{v}-\gamma^{0} V_{s}\right) \gamma^{D+1}\right\} K_{D} \Psi_{\kappa_{D}} \\
& =\kappa_{D} r^{-\frac{D-1}{2}}\left\{-\left(\partial_{r}+\kappa_{D} \gamma^{0}\right) \Phi_{\kappa_{D}}\right. \\
& \left.+\left(V_{v}-\gamma^{0} V_{s}\right) \Theta_{\kappa_{D}}\right\}
\end{aligned}
$$


and

$$
\begin{aligned}
\gamma^{D+1} \gamma^{0} \gamma^{i} \frac{x^{i}}{r}\left(\alpha_{v} m+\alpha_{s} H\right) \Psi_{\kappa_{D}} \\
\quad=-r^{-\frac{D-1}{2}}\left\{\alpha_{s}\left(\partial_{r}-\frac{\kappa_{D}}{r} \gamma^{0}\right) \Theta_{\kappa_{D}}\right. \\
\left.+\left[\alpha_{s} \gamma^{0}\left(m+V_{s}+\gamma^{0} V_{v}\right)+m \alpha_{v}\right] \Phi_{\kappa_{D}}\right\} .
\end{aligned}
$$

Thus, the explicit form of the Lippmann-Johnson operator $B$ acting on an eigenstate $\Psi_{\kappa_{D}}$ of the Hamiltonian $H$ is

$$
\begin{array}{r}
B \Psi_{\kappa_{D}}=-r^{-\frac{D-1}{2}\left\{\left(\alpha_{s} \partial_{r}-\kappa_{D} V_{v}\right) \Theta_{\kappa_{D}}\right.}+\left(\kappa_{D}\left(\partial_{r}+\frac{\kappa_{D}}{r} \gamma^{0}\right)+m \alpha_{v}\right) \Phi_{\kappa_{D}} \\
\left.+\alpha_{s} \gamma^{0}\left(m+V_{s}+\gamma^{0} V_{v}\right) \Phi_{\kappa_{D}}\right\} .
\end{array}
$$

\section{Calculation of $B^{2}$}

With the definitions $A_{1}=H-\gamma^{0} m$ and $A_{2}=\alpha_{v} m+\alpha_{s} H$, we find the following commutation relations

$$
\begin{aligned}
{\left[A_{1}, A_{2}\right] } & =-2 m \alpha_{S} \gamma^{i} p^{i}, \\
{\left[A_{1}, \gamma^{D+1}\right] } & =-2 \gamma^{D+1} \gamma^{0} V_{s}, \\
{\left[A_{2}, \gamma^{D+1}\right] } & =-2 \alpha_{s} \gamma^{D+1} \gamma^{0}\left(m+V_{s}\right), \\
{\left[A_{1}, \gamma^{0} \gamma^{i} \frac{x^{i}}{r}\right] } & =\frac{2 i \gamma^{0}}{r} K_{D}+2 V_{s} \gamma^{i} \frac{x^{i}}{r}, \\
{\left[A_{2}, \gamma^{0} \gamma^{i} \frac{x^{i}}{r}\right] } & =2 i V_{s} \gamma^{0} K_{D}+2 \alpha_{s}\left(m+V_{s}\right) \gamma^{i} \frac{x^{i}}{r} .
\end{aligned}
$$

Since $\left[K_{D}, \gamma^{0} \gamma^{i} \frac{x^{i}}{r}\right]=0$ and $\left\{K_{D}, \gamma^{D+1}\right\}=0$, and because of the antihermiticity of $\gamma^{j}(j=1,2, \ldots, D)$, we finally obtain

$$
\begin{aligned}
B^{2} & =B^{\dagger} B=A_{1}^{2} K_{D}^{2}+A_{2}^{2}-i A_{1} \gamma^{0} \gamma^{i} \frac{x^{i}}{r} A_{2} K_{D} \\
& +i A_{2} \gamma^{0} \gamma^{i} \frac{x^{i}}{r} A_{1} K_{D} \\
& =A_{1}^{2} K_{D}^{2}+A_{2}^{2}+2 m\left(V_{v}+\gamma^{0} V_{s}\right) \gamma^{0} K_{D}^{2} \\
& =\left(H^{2}-m^{2}\right) K_{D}^{2}+A_{2}^{2} \\
& =\left(\alpha_{v} m+\alpha_{s} H\right)^{2}+K_{D}^{2}\left(H^{2}-m^{2}\right) .
\end{aligned}
$$




\section{Acknowledgments}

This work was partially supported by SNI-México, COFAA-IPN, EDI-IPN, SIP-IPN project number 20110127, and ADI-UACM project number 7DA2023001.

\section{References}

[1] Greiner W., Relativistic Quantum Mechanics (Springer-Verlag, Berlin) 2000.

[2] Tutik, R. S. J. Phys. A: Math. and Gen., 25 (1992) L413.

[3] Junker G., Supersymmetric Methods in Quantum and Statistical Physics (Springer-Verlag, New York) 1996.

[4] Cooper F., Khare A. and Sukhatme U., Supersymmetry in Quantum Mechanics (World-Scientific, Singapure) 2001.

[5] Hecht K.T., Quantum Mechanics (Springer-Verlag, New York) 2000.

[6] Sukumar C.V., J. Phys. A: Math. Gen., 18 (1985) L697.

[7] Dahl J.P. and Jorgensen T., Int. J. Quantum Chem., 53 (1995) 161.

[8] Salazar-Ramírez M. et. al., J. Phys. A: Math. Theor., 43 (2010) 445203.

[9] Greiner, W., Müller B. and Rafelski J., Quantum Electrodymanics of Strong Fields (Springer-Verlag, Berlin) 1985.

[10] Guo-Xing J. and Zhong-Zhou R, Commun. Theor. Phys., 49 (2008) 319.

[11] Leviatan, A., Phys. Rev. Lett., 92 (2004) 20.

[12] A. Joseph, Rev. Mod. Phys., 39 (1967) 829.

[13] Katsura H. and Aoki H., J. Math. Phys., 47 (2006) 032301.

[14] Dong S.H., Sung G.H. and Popov D., J. Math. Phys., 44 (2003) 4467.

[15] Salazar-Ramírez M. et. al., Eur. Phys. Lett., 95 (2011) 60002.

[16] Ehrenfest P., Ann. Phys., 61, (1920) 440. 
[17] Swiebach B., A First Course in String Theory, (Cambridge University Press, UK) 2009.

[18] Braga N. R. F. and D'Andrea R., arXiv:quant-ph/0511078v1

[19] Johnson M.H. and Lippmann B.A., Phys. Rev. 78 (1950) 329A.

[20] Tangerman D.R. and Tgon J.A., Phys. Rev. A, 48 (1993) 1089.

[21] Sakurai J.J., Advanced Quantum Mechanics (Addison-Wesley, Reading MA) 1967.

[22] Thaller B., Advanced Visual Quantum Mechanics (Springer, USA) 2005.

[23] Chen J.L. Deng D.L. and Hu M.G., Phys. Rev. A, 77 (2008) 034102. 